\title{
ANÁLISE COMPUTACIONAL DOS MODOS E FREQUÊNCIAS NATURAIS DE VIBRAÇÃO DE UMA VIGA ENGASTADA-LIVRE
}

\section{COMPUTATIONAL ANALYSIS OF NATURAL FREQUENCIES AND MODES OF VIBRATION OF A CLAMPED-FREE BEAM}

Filipe Bittencourt Figueiredo ${ }^{1}$, Guilherme Crepaldi Camarini ${ }^{1}$, Daniele Araujo Altran ${ }^{2}$, Cássio Fabian Sarquis de Campos ${ }^{2}$

${ }^{1}$ Discente, ${ }^{2}$ Docente da Faculdade de Engenharia da Universidade do Oeste Paulista (UNOESTE), Presidente Prudente, SP. (cassio@unoeste.br)

RESUMO - Este trabalho teve como objetivo realizar a modelagem teórica e computacional de uma viga engastada-livre, mostrando os quatro primeiros modos e suas respectivas frequências naturais de vibração. O estudo da dinâmica de estruturas sejam elas rígidas ou flexíveis, sem a ação de forças externas ou sob a ação destas, tais como, ondas regulares marítimas, ventos, vibrações mecânicas externas de qualquer ordem, é de vital importância para a prevenção de possíveis problemas estruturais, tais como trincas, deslocamentos, rupturas parciais ou totais dessas estruturas. O estudo da dinâmica da viga foi, inicialmente, realizado através da abordagem Euler-Bernoulli e a flexibilidade foram estudadas a partir do método dos Modos Assumidos. Os recursos computacionais disponíveis contou com o Matlab, para implementação encontrando os modos de vibração e suas respectivas frequências naturais e o Maple, empregado para implementar os modos de vibração tridimensionalmente e desenvolver os cálculos diferenciais do trabalho. Durante a implementação computacional optou-se por fazer com que os programas desenvolvidos permitam novos estudos com diferentes tipos de vigas, vínculos e propriedades físicas. Além de obter os modos e as frequências naturais de vibração, este trabalho proporcionou ainda, o desenvolvimento de habilidades para modelar matematicamente a dinâmica de estruturas bem como, habilidades de implementar modelos através de programações. Para projetos futuros indica-se resolver as equações de modo a considerar forças externas atuantes no sistema, a fim de se estudar o movimento da viga e sua flexibilidade estrutural quando sujeitas às fontes de excitações externas.

Palavras-chave: dinâmica; estruturas; viga; engastada-livre; modos assumidos.

ABSTRACT - This study aimed to perform theoretical and computational modeling of a clamped-free, showing the first four vibration modes and their natural frequencies. The study of dynamic structures whether rigid or flexible, without the action of external forces or under the action of these, such as regular sea waves, wind, 
mechanical vibration of external order, is of vital importance for the prevention of possible problems structural, such as cracks, displacement, partial or total disruption of these structures. The study of the dynamics of the beam was initially performed by Euler-Bernoulli approach and flexibility has been studied from the assumed modes method. The computational resources available had the Matlab, to implement finding the vibration modes and their natural frequencies, the Maple was used to implement the three-dimensional vibration modes and develop differential calculations work. During the computational implementation chose to make the programs developed will allow further studies with different types of beams and physical properties. This work provided further skill development to mathematically model the dynamics of structures as well as skills to implement models through programming. For future plans indicates to solve the equations in order to consider external forces acting on the system, in order to study the motion of the beam and its structural flexibility when subjected to external sources of excitations.

Key-words: dynamics; structures; beam; clamped-free; assumed modes. 


\section{INTRODUÇÃO}

A engenharia de estruturas é uma área de grande importância na construção civil, uma vez que sua essência é a otimização de projetos. As estruturas em geral são constituídas por elementos estruturais, por meio dos quais, as cargas são transmitidas, desde o topo das edificações até as fundações. Entre os elementos geralmente utilizados, destacam-se as lajes, as vigas e os pilares. Em especial, o estudo de vigas é de fundamental importância, tendo em vista sua função estrutural. O estudo das respostas estáticas e dinâmicas de componentes estruturais, tais como as vigas, sob a ação de várias condições de carga, é muito útil para a modelagem e análise do comportamento de estruturas mais reais e complexas sujeitas a carregamentos similares.

Neste contexto, a análise estrutural dinâmica completa tem por finalidade quantificar os esforços internos, as velocidades, as acelerações, os deslocamentos, frequências e modos de vibração, que são desenvolvidos em qualquer sistema estrutural, quando o mesmo é submetido a um carregamento arbitrário, cuja intensidade, direção e sentido variem com o tempo (RODRIGUES, 1997).

$\mathrm{Na}$ dinâmica de estruturas, uma grande preocupação, é ocorrência de vibrações excessivas, indesejáveis do ponto de vista estrutural, já que podem causar danos na estrutura devido ao alto valor dos esforços solicitantes provocados. As vibrações também podem ocasionar a fadiga dos elementos estruturais, além de provocar desconforto nos indivíduos que utilizam a estrutura regularmente, além de, comprometer sua segurança (LARA, 2007).

Desse modo, desde a disseminação da informática ocorrida no final da década de 1980, são promovidos muitos avanços tecnológicos, com grande aumento da capacidade de armazenamento, gerenciamento e processamento de dados apresentados pelos computadores de pequeno porte. Logo, o engenheiro de estruturas passou a ter acesso a equipamentos e programas computacionais que possibilitam uma análise estrutural baseada em modelos mais refinados, proporcionando um aumento da confiabilidade e diminuição de custos dos projetos e das construções (WAIDEMAN, 2004).

Neste cenário, as ferramentas computacionais, baseadas em métodos numéricos, são de grande importância, pois, auxiliam os profissionais a obterem, com maior agilidade, projetos de alta qualidade, diminuindo custos e prazos (TIMOSHENKO; GOODIER, 1934).

Normalmente, a utilização de modelos numéricos está associada à concepção e projeto de novas estruturas ou 
então a atividades relacionadas com 0 acompanhamento e/ou a avaliação de segurança de estruturas existentes que apresentem um risco potencial significativo (MENDES; OLIVEIRA, 2008).

\section{JUSTIFICATIVA}

O estudo da dinâmica de estruturas sejam elas rígidas ou flexíveis sob a ação de forças externas ou não, tais como, ondas regulares marítimas, ventos, vibrações mecânicas externas de qualquer ordem, é de vital importância para a prevenção de possíveis problemas estruturais, tais como trincas, deslocamentos, rupturas parciais ou totais dessas estruturas.

Para tanto, faz-se antes necessário conhecer os modos e as frequências naturais de vibração da estrutura. Normalmente, estes estudos são realizados através de modelos matemáticos computacionais.

Especificamente, a realização deste trabalho dá início às atividades de pesquisas na área de dinâmica estrutural e modelagem computacional de sistemas dinâmicos na Faculdade de Engenharia "Conselheiro Algacyr Munhoz Maeder" de Presidente Prudente - UNOESTE/SP.

\section{OBJETIVO}

\footnotetext{
Em estudos sobre dinâmica de estruturas é fundamental obter as frequências naturais e os modos de vibração
}

de uma viga engastada-livre garantindo assim, o estudo da flexibilidade natural do sistema, sem ação de forças externas. Logo, este trabalho teve como objetivo realizar a modelagem matemática e computacional de uma viga engastada-livre, descrevendo os quatro primeiros modos de vibração e obtendo suas respectivas frequências naturais de vibração.

As propriedades físicas e as condições de contorno são abordadas de forma genérica de modo que, o modelo computacional desenvolvido possa ser aplicado a estudos de outros tipos de vínculos estruturais com dimensões e propriedades físicas diferentes.

\section{METODOLOGIA}

Inicialmente, buscou-se na literatura conhecimentos teóricos que permitiram descrever a flexibilidade de uma viga engastada-livre e a partir deste, estudar o comportamento de uma estrutura hipotética.

O sistema modelado é composto por uma barra de comoprimento $L$, com área de seção transversal $A$ e massa $M$ engastada em uma de suas extremidades a um corpo rígido e a outra extremidade considerada livre, conforme ilustrado a seguir. 
esforços. Dentre estes, optou-se pelo método conhecido como método de EulerBernoulli, onde a vibração transversal é governada pela seguinte equação diferencial.

Figura 1. Viga engastada-livre.

Fonte: Autores.

Meirovitch (1975) e Craig (1981)

mostram muitos métodos de se modelar uma viga sujeita a vários tipos de vínculos e

$$
\frac{\partial^{2}}{\partial x^{2}}\left(E . I . \frac{\partial^{2} y(x, t)}{\partial x^{2}}\right)+\rho . A\left(\frac{\partial^{2} y(x, t)}{\partial t^{2}}\right)=Q(x, t)
$$

esforços causados por ondas regulares

$\mathrm{Na}$ equação (1), $E$ representa o módulo de elasticidade da viga (Pa), I o momento de inércia da viga $\left(\mathrm{m}^{4}\right), \rho$ massa por unidade de volume $\left(\mathrm{Kg} / \mathrm{m}^{3}\right), A$ como área da seção transversal $\left(m^{2}\right), y(x, t)$ representa o deslocamento flexível como função do comprimento $L(\mathrm{~m})$ da viga e do tempo $t(\mathrm{~s})$ e $Q(x, t)$ representa uma perturbação externa atuante sobre a viga, como por exemplo,

$$
\frac{\partial^{2}}{\partial x^{2}}\left(E . I . \frac{\partial^{2} y(x, t)}{\partial x^{2}}\right)+\rho . A\left(\frac{\partial^{2} y(x, t)}{\partial t^{2}}\right)=0
$$

O termo $y(x, t)$ na equação (2), refere-se a flexibilidade da viga variando tanto ao longo do comprimento $L$, quanto no tempo t. Para estudar esta flexibilidade escolheu-se o método dos Modos Assumidos.

Este método modela a deflexão de estruturas como uma série finita de funções, marítimas, ventos ou vibrações mecânicas externas de qualquer ordem.

Como neste trabalho, buscou-se os modos de vibração e suas respectivas frequências de vibração para uma viga que oscila livremente, assumiu-se que não há esforço externo, o que implica em fazer $Q(x, t)=0$. Desta forma, a equação a equação (1) torna-se:

dependentes do espaço, multiplicadas por funções dependentes do tempo (JUNKINS; KIM, 1993).

Campos (2001) mostra que as funções dependentes do espaço constituem um conjunto de funções linearmente independentes selecionadas para satisfazer 
as condições geométricas, sendo chamadas de funções admissíveis. Se a formulação do problema exigir que, além das condições geométricas, todas ou algumas das condições físicas sejam atendidas, novas funções devem ser tomadas e estas passam a ser chamadas de funções comparação. As condições geométricas estão relacionadas com a

$$
y(x, t)=\Phi(x) \cdot \Psi(t)
$$

Onde, $\Phi(x)$ denota as funções de forma escolhidas para descrever as deflexões das vigas ao longo de seu comprimento e

$$
\Psi(t)=\cos (\omega . t)
$$

Onde, $\omega$ corresponde a frequência angular $(\mathrm{rad} / \mathrm{s})$ e t corresponde ao tempo (s)

$$
y(x, t)=\Phi(x) \cdot \cos (\omega \cdot t)
$$

Substituindo a equação (5) em (2) e resolvendo, obtém-se a equação:

$$
E . I \frac{\partial^{2}}{\partial x^{2}}\left(\frac{\partial^{2} \Phi(x)}{\partial x^{2}}\right)-\rho . A \omega^{2} \Phi(x)=0
$$$$
\frac{\partial^{4} \Phi(x)}{\partial x^{4}}=\lambda^{4} . \Phi(x)
$$

geometria da viga nas extremidades, enquanto que, as condições físicas consideram a presença de tensões de cisalhamento e momento fletor na viga.

Matematicamente, segundo Junkins e Kim (1993) e Meirovitch (1975), pode-se escrever a solução dos movimentos flexíveis na seguinte forma:

$\Psi(t)$ corresponde a função do movimento dependente do tempo, onde esta última pode ser expressa por:

(HALLIDAY; RESNICK; WALKER, 2010). Assim, substituindo (4) em (3), obtém-se:

Pode-se escrever a equação anterior para o movimento de vibração livre de uma viga uniforme da seguinte forma: 
Onde, por simplificação o termo $\lambda$

$$
\lambda^{4}=\frac{\rho A \omega^{2}}{E . I}
$$

pode ser escrito como:

A solução da Equação (7) é dada por Young e Felgar (1949) e dada por:

$$
\Phi(x)=C_{1} \operatorname{senh}(\lambda \cdot x)+C_{2} \cosh (\lambda \cdot x)+C_{3} \operatorname{sen}(\lambda \cdot x)+C_{4} \cos (\lambda \cdot x)
$$

Onde, $C_{1}, C_{2}, C_{3}, C_{4}$ são constantes de amplitude e $\lambda$ corresponde ao autovalor da função.

Segundo Thonson (1981) as condições de contorno para uma viga engastada-livre são dadas de acordo com a Tabela 1.

Tabela 1. Condições de contorno para uma viga engastada-livre.

\begin{tabular}{cl}
\hline Na extremidade fixa $(\mathrm{x}=0)$ & $\begin{array}{l}\mathrm{Na} \\
\text { Extremidade } \\
\text { livre }(\mathrm{x}=\mathrm{L})\end{array}$ \\
\hline$\Phi(x)=0$ & $\frac{d^{2} \Phi(x)}{d x^{2}}=0$ \\
\hline$\frac{d \Phi(x)}{d x}=0$ & $\frac{d^{3} \Phi(x)}{d x^{3}}=0$ \\
\hline
\end{tabular}

A Tabela (1) mostra as condições de contornos para as extremidades fixa e livre. Ao interpretar as condições de contorno acima, pode-se dizer que, o deslocamento e a curvatura devido a forças externas são nulos na extremidade fixa; já na extremidade livre mostram que o momento fletor e o cortante são nulos neste ponto.

Aplicando as condições de contorno da Tabela 1, na expressão (9) obtém-se o seguinte conjunto de equações diferenciais:

Fonte: (TIMOSHENKO; GOODIER, 1934)

Nota: Dados trabalhados pelos autores.

$$
\begin{aligned}
& \Phi(x)=0 \\
& \frac{d \Phi(x)}{d x}=\lambda\left(C_{1} \cosh (\lambda \cdot x)+C_{2} \operatorname{senh}(\lambda \cdot x)+C_{3} \cos (\lambda \cdot x)-C_{4} \operatorname{sen}(\lambda \cdot x)\right) \\
& \frac{d^{2} \Phi(x)}{d x^{2}}=\lambda^{2}\left(C_{1} \operatorname{senh}(\lambda \cdot x)+C_{2} \cosh (\lambda \cdot x)-C_{3} \operatorname{sen}(\lambda \cdot x)-C_{4} \cos (\lambda \cdot x)\right) \\
& \frac{d^{3} \Phi(x)}{d x^{3}}=\lambda^{3}\left(C_{1} \cosh (\lambda \cdot x)+C_{2} \operatorname{senh}(\lambda \cdot x)-C_{3} \cos (\lambda \cdot x)+C_{4} \operatorname{sen}(\lambda \cdot x)\right)
\end{aligned}
$$


Pode-se escrever as equações (10), (11), (12) e (13) na forma de determinante.

$$
\left[\begin{array}{cccc}
0 & 1 & 0 & 1 \\
\lambda & 0 & \lambda & 0 \\
\lambda^{2} \operatorname{senh}(\lambda L) & \lambda^{2} \cosh (\lambda L) & -\lambda^{2} \operatorname{sen}(\lambda L) & -\lambda^{2} \cos (\lambda L) \\
\lambda^{3} \cosh (\lambda L) & \lambda^{3} \operatorname{senh}(\lambda L) & -\lambda^{3} \cos (\lambda L) & \lambda^{3} \operatorname{senh}(\lambda L)
\end{array}\right] \cdot\left[\begin{array}{l}
C_{1} \\
C_{2} \\
C_{3} \\
C_{4}
\end{array}\right]=\left[\begin{array}{l}
0 \\
0 \\
0 \\
0
\end{array}\right]
$$

Observando o determinante anterior nota-se que deve server nulo devido as condições de contorno do tipo de viga

$$
\cosh (\lambda L) \cdot \cos (\lambda L)=-1
$$

Ainda segundo Teixeira (2002), a solução da equação (15) não é trivial e o conjunto de suas raizes são os autovalores $\lambda_{i}$ multiplicados pelo comprimento $L$ da viga. Estes valores são apresentados na Tabela (2).

Tabela 2. Valores de $\lambda_{i} L$ para os quatro primeiros modos.

\begin{tabular}{l|c}
\hline \multicolumn{2}{|c}{$\lambda_{i} L$} \\
\hline$\lambda_{1} L$ & 1,8751 \\
\hline$\lambda_{2} L$ & 4,6941 \\
\hline
\end{tabular}

$$
\omega_{i}=\frac{\left(\lambda_{i} L\right)^{2}}{L^{2}} \cdot\left(\frac{E . I}{\rho . A}\right)^{1 / 2}
$$

Utilizando os valores da Tabela (2) na equação (16), obtém-se as frequência de

$$
\omega_{1}=\frac{3,5171}{L^{2}} \cdot\left(\frac{E . I}{\rho . A}\right)^{1 / 2}
$$

adotada (TEIXEIRA, 2002). Assim, obtém-se a equação característica deste sistema, dada por:

$$
\text { Lembrando que, } \quad \lambda^{4}=\frac{\rho A . \omega^{2}}{E . I}
$$
fornecem as frequências angulares $\omega$ de vibração livre, pode-se rearranjar esta equação de forma equacionar uma fomra de se obter as frequências naturais de vibração. Assim,

vibração da viga para os quatro primeiros modos de vibração, dadas por: 


$$
\begin{aligned}
& \omega_{2}=\frac{22,0345}{L^{2}} \cdot\left(\frac{E . I}{\rho . A}\right)^{1 / 2} \\
& \omega_{3}=\frac{61,6978}{L^{2}} \cdot\left(\frac{E \cdot I}{\rho . A}\right)^{1 / 2} \\
& \omega_{4}=\frac{120,9010}{L^{2}} \cdot\left(\frac{E . I}{\rho . A}\right)^{1 / 2}
\end{aligned}
$$

tanto, utiliza-se as duas primeiras equações

Uma vez, obtidas as equações das frequências naturais de vibração, pode-se agora determinar os modos de vibração. Para

$$
\begin{aligned}
& C_{4}=-C_{2} \\
& C_{3}=-C_{1}
\end{aligned}
$$

Observando a terceira equação do determinante estabelece-se que:

$C_{1} \operatorname{senh}(\lambda . L)+C_{2} \cosh (\lambda . L)-C_{3} \operatorname{sen}(\lambda . L)-C_{4} \cos (\lambda . L)=0$

Combinando as equações (21), (22) e (23), obtém-se:

$C_{1} \operatorname{senh}(\lambda . L)+C_{2} \cosh (\lambda . L)+C_{1} \operatorname{sen}(\lambda . L)+C_{2} \cos (\lambda . L)=0$

Separando os termos $C_{1}$ e $C_{2}$ na equação (24) têm-se:

$$
C_{1}=-\left[\frac{\cosh (\lambda . L)+\cos (\lambda . L)}{\operatorname{senh}(\lambda . L)+\operatorname{sen}(\lambda . L)}\right] \cdot C_{2}
$$

Utilizando as condições de contorno e escrever a função de forma para o simplifciando a equação (25), pôde-se como:

$$
\Phi_{i}(x)=\cosh (\lambda . x)-\cos (\lambda . x)-\sigma_{i}[\operatorname{senh}(\lambda . x)-\operatorname{sen}(\lambda . x)]
$$
característicos do tipo de viga considerada no

Onde, $x$ corresponde ao sistema . comprime nto da viga considerada, $\lambda_{i}$ constitui uma valores relacionados à frequência natural de vibração do $i$-ésimo modo e $\sigma_{i}$ corresponde a valores

\section{RESULTADOS}

A função de forma obtida neste trabalho representando os deslocamentos 
flexíveis do sistema ao longo de seu comprimento é então dada por:

$$
\Phi_{i}(x)=\cosh \left(\lambda_{i} \cdot x\right)-\cos \left(\lambda_{i} \cdot x\right)-\sigma_{i} \cdot\left[\operatorname{senh}\left(\lambda_{i} \cdot x\right)-\operatorname{sen}\left(\lambda_{i} \cdot x\right)\right]
$$

A equação (27) concorda com os resultados apresentados por Young \& Felgar Jr. (1949), onde também fornecem os valores de $\lambda_{i}$ e $\sigma_{i}$ para este tipo de viga, apresentados na Tabela (3). Onde, i é corresponde ao $i$-ésimo modo de vibrar.

Tabela 3. Valores de $\lambda$ e $\sigma$.

\begin{tabular}{|c|c|c|c|c|}
\hline $\begin{array}{l}\text { Modo } \\
\text { s }\end{array}$ & 10 & 20 & 30 & 40 \\
\hline \multirow[t]{2}{*}{$\lambda$} & 1,875 & 4,694 & 7,854 & 10,995 \\
\hline & 1 & 0 & 7 & 5 \\
\hline \multirow[t]{2}{*}{$\sigma$} & 0,734 & 1,018 & 0,999 & 1 \\
\hline & 1 & 5 & 2 & \\
\hline
\end{tabular}

Fonte: (YOUNG; FELGAR JR, 1949).

Nota: Dados trabalhados pelos autores.

Para modelagem computacional, escolheu-se um modelo aleatório de viga de concreto. As especificações da viga proposta encontram-se dispostas na Tabela (4).
Tabela 4. Especificações da viga.

\begin{tabular}{lccl}
\hline \multicolumn{2}{l}{ Propriedades } & Símbolo & Valor \\
\hline $\begin{array}{l}\text { Massa específica } \\
\text { volumétrica } \\
\text { concreto }\end{array}$ & $\rho$ & $2400 \mathrm{Kg} / \mathrm{m}^{3}$ \\
\hline $\begin{array}{l}\text { Massa total } \\
\text { Área de seção }\end{array}$ & $A$ & $0,0225 \mathrm{~m}^{2}$ \\
$\begin{array}{l}\text { tranversal } \\
\text { (0,15mx0,15m) }\end{array}$ & & \\
\hline $\begin{array}{l}\text { Comprimento } \\
\text { Módulo de }\end{array}$ & $E$ & $\begin{array}{l}30641,97 \\
\mathrm{MPa}\end{array}$ \\
\hline $\begin{array}{l}\text { Elasticidade } \\
\text { Momento de Inércia }\end{array}$ & $I$ & $\begin{array}{l}8,64.10^{-6} \\
\mathrm{~m}^{4}\end{array}$ \\
\hline
\end{tabular}

Fonte: (BEER; JOHSNTON JR., 1995).

Nota: Dados trabalhados pelos autores.

Tomando-se a equação (27) e as especificações das Tabelas (3) e (4), foi possível a partir do software Matlab obter os quatro primeiros modos de vibração da viga engastada-livre representados nos seguintes gráficos da Figura (2). 

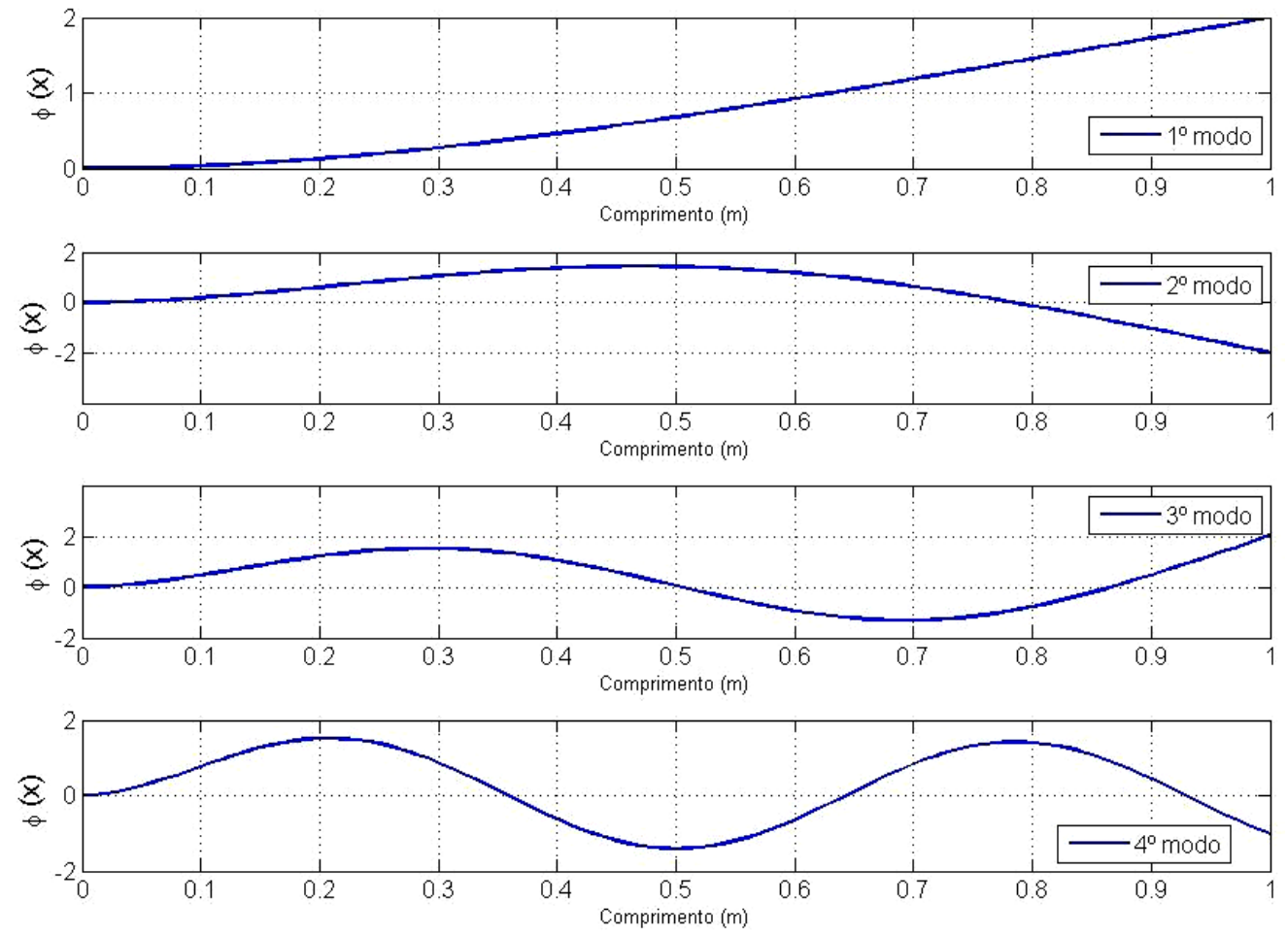

Figura 2. Forma para os quatro primeiros modos de vibração $(l=1,0 m)$.

Na Figura (2), o eixo y representa a função $\Phi(x)$ como modo de mostrar o deslocamento flexível ao longo do comprimento da viga.

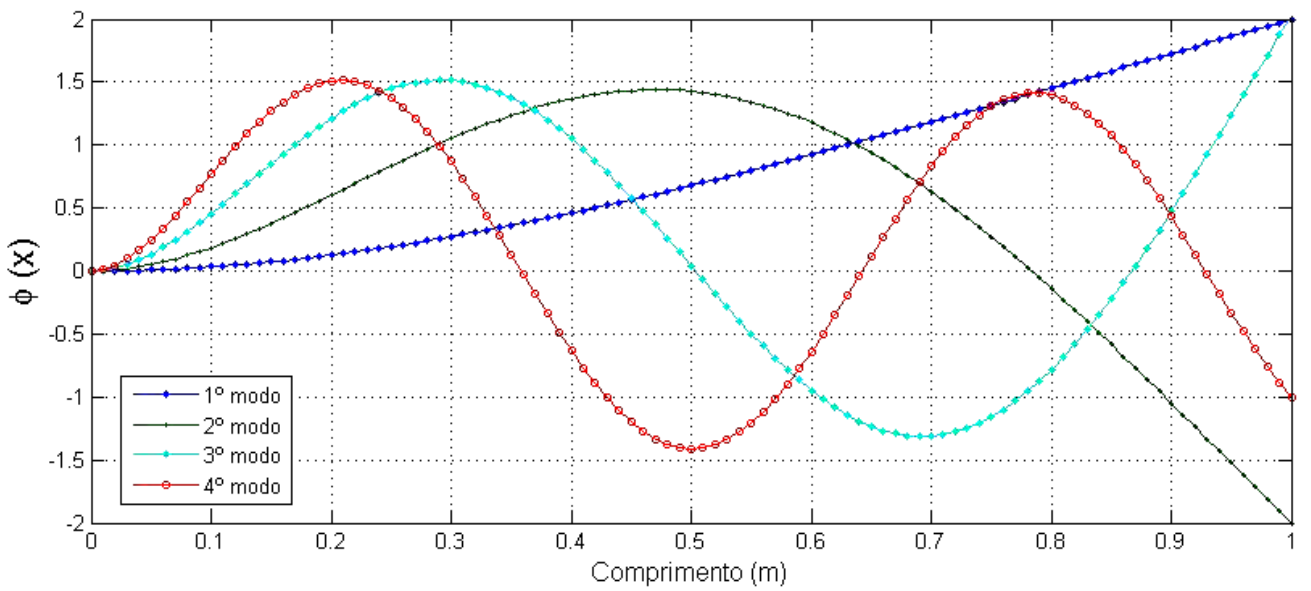

Figura 3. Os quatro primeiros modos de vibração.

Com o intuito de proprorcionar um maior entendimento das funções de forma e visualização dos modos naturais de vibração, fez-se uma representação espacial destes modos quando oscila livremente
A Figura (3) ilustra os quatro primeiros modos de vibração um único gráfico. 

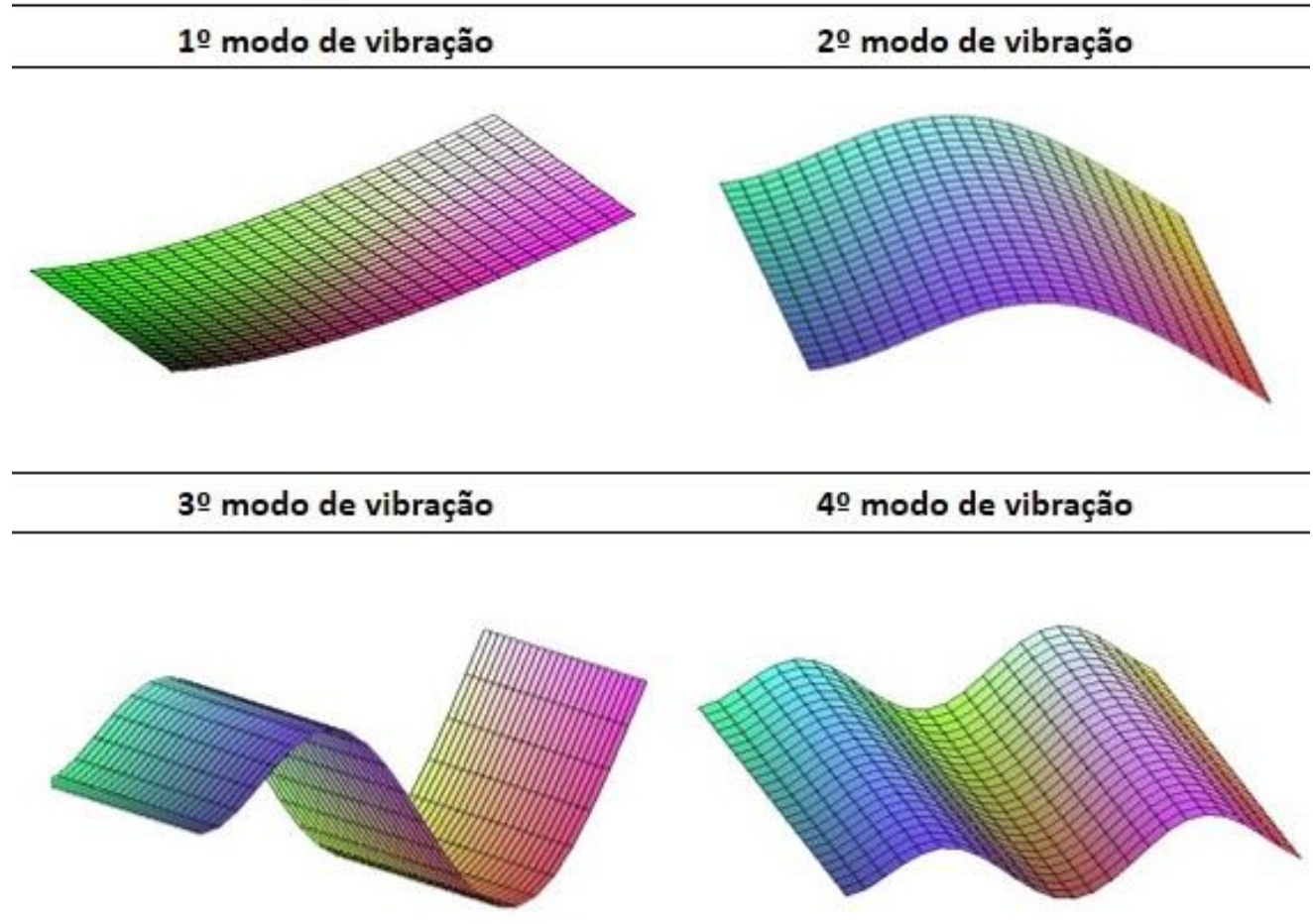

Figura 4. Modos de vibração espacialmente representados.

A Figura (4) ilustra os quatro primeiros modos de vibração da viga em uma visão tridimensional.

As frequências de vibração da viga proposta foram calculadas a partir das equações (17), (18), (19) e (20) e os resultados encontram-se na Tabela (5).

Tabela 5 - Frequências naturais de vibração.

\begin{tabular}{|c|c|c|}
\hline Modo & $\begin{array}{c}\text { Frequência } \\
\text { natural (rad/s) }\end{array}$ & $\begin{array}{l}\text { Frequência } \\
\text { natural }(\mathrm{Hz})\end{array}$ \\
\hline 10 & 7,7875 & 1,2394 \\
\hline 20 & 48,7889 & 7,7649 \\
\hline 30 & 136,6117 & 21,7423 \\
\hline 40 & 267,6999 & 42,6056 \\
\hline
\end{tabular}

Nota: Dados trabalhados pelos autores.
A Tabela (5) mostra as frequências naturais de vibração da viga engastada-livre proposta, sendo estas, únicas para cada tipo de material uma vez que, leva-se em consideração a massa específica, comprimento, o módulo de elasticidade, o momento de inércia e consequentemente a geometria da viga (THONSON, 1981).

\section{DISCUSSÃO}

Conhecer as frequências naturais de vibração de uma estrutura é de vital importância, pois caso uma fonte externa excite a estrutura na mesma frequência, poderá ocorrer o fenômeno de ressonância que poderá levar ao aumento da amplitude 
de vibração estrutural e consequentemente a maiores deformações promovendo fissuras, trincas, rupturas ou danos maiores.

Desta forma, durante o projeto de uma estrutura sugere-se ao engenheiro que o faça, levando-se também em consideração nos estudos, o cálculo das frequências naturais de vibração de modo que, possíveis fontes externas de vibração não atinjam tais frequências. Logo, a absorção de energia dessas fontes externas e consequentemente, - aumento da amplitude de vibração poderão não ocorrer, assim, danos ou colapsos da estrutura serão evitados.

\section{CONCLUSÃO}

Este trabalho apresentou um estudo da dinâmica de uma viga engastada-livre considerando apenas vibração livre. Este foi o primeiro estudo de dinâmica das estruturas realizado na Faculdade de Engenharia de Presidente Prudente-SP.

Para encontrar os modos de vibração e suas respectivas frequências naturais utilizou-se o método de Euler-Bernoulli e a flexibilidade foi modelada pelo método dos modos assumidos. Os resultados para os modos de vibração da estrutura ao longo de seu comprimento foram estudados pela função correspondente à variação espacial deste método.

O objetivo de se obter os modos de vibração para o entendimento deste fenômeno intrinsico da estrutua não só foi cumprido através do software Matlab , como também, foi acrescido por representações tridimensionais no software Maple ${ }^{\oplus}$. Desta forma, o entendimento dos modos de vibração ficou mais claro e mais fácil de ser compreendido.

As frequências de vibração foram obtidas a partir da função que descreve o movimento oscilatório da viga segundo a função temporal presente no método dos modos assumidos.

$\mathrm{Na}$ implementação computacional tanto dos modos de vibração quanto nos cálculos das frequências de vibração, houve a preocupação em deixar as entradas dos programas disponíveis para qualquer tipo de estrutura, vínculos e propriedades físicas, tais como, comprimento da viga, área de seção, massa específica, módulo de elasticidade, momento de inércia e tipos de funções assumidas, para que futuros estudos com outros tipos de estruturas possam fazer uso dos programas.

Nesse trabalho, pôde se aplicar dois softwares de modelagem computacional, proporcionando o desenvolvimento de habilidades para modelar matematicamente a dinâmica de estruturas bem como, de implementar modelos através de programações.

Indica-se para projetos futuros resolver a equação de Euler-Bernoulli sob 
ação de forças externas, a fim de se estudar o movimento da viga, e sua flexibilidade estrutural quando sujeitas às excitações externas.

\section{REFERÊNCIAS}

BEER, F.P.; JOHSNTON JR., E.R. Resistência dos materiais. 3. ed. São Paulo: Makron, 1995.

CAMPOS, C.F.S. Dinâmica e constrole de movimento de corpo rígido de um manipulador robótico rígido flexível. 2001. 107f. Dissertação (Mestrado em Engenharia e Tecnologia Espaciais) - Instituto Nacional de Pesquisas Espaciais, INPE, São José dos Campos.

CRAIG, R.R. Structural dynamics. 1. ed. New York: John Wiley, 1981.

HALLIDAY, D.; RESNICK, R.; WALKER, J. Fundamentos da física. Rio de Janeiro: Livros Técnicos e Científicos, 2010. vol. 2.

JUNKINS, J.L.; KIM, Y. Introduction to dynamics and control of flexible structures. Washington: American Institute of Aeronautics and Astronautics, 1993. http://dx.doi.org/10.2514/4.862076

LARA, V. Controle de vibrações em vigas metálicas com distintas configurações de apoio utilizando amortecedores de massa sintonizados. 2007. 156f. Dissertação (Mestrado em Engenharia Civil) Departamento de Engenharia Civil e Ambiental, Universidade de Brasília, Brasília.

MEIROVITCH, L. Elements of vibration analysis. Tokyo: McGraw-Hill, 1975.

MEIROVITCH, L. Computational methods in structural dynamics. Maryland: Sijthoff \& Noordhoff, 1980.
MENDES, P.; OLIVEIRA, S. Análise dinâmica de estruturas: utilização integrada de modelos de identificação modal e modelos de elementos finitos. Lisboa: Laboratório Nacional de Engenharia Civil, 2008.

RODRIGUES, R.O. Análise dinâmica bidimensional não-linear física e geométrica de treliças de aço e pórticos de concreto armado. 1997. 275f Tese (Doutorado em Engenharia Civil) - Escola de Engenharia de São Carlos, Universidade de São Paulo, São Carlos.

TEIXEIRA, G.M. Análise de impacto em vigas usando-se o método de integração explícita no tempo. 2002. 142f. Dissertação (Mestrado em Engenharia Civil) - Escola de Engenharia de São Carlos, Universidade de São Paulo, São Carlos.

TIMOSHENKO, S.; GOODIER, J.N. Theory of elasticity. Tokyo: McGraw-Hill, 1934.

THONSON, W.T. Theory of Vibration with applications. New Jersey: Prentice Hall, 1981.

WAIDEMAN, L. Análise dinâmica de placas delgadas utilizando elementos finitos triangulares e retangulares. 2004. 153f. Dissertação (Mestrado em Engenharia) Faculdade de Engenharia, Universidade Estadual Paulista, Ilha Solteira.

YOUNG, D.; FELGAR JR, P. Tables of characteristic functions representing normal modes of vibration of a beam. Texas: The University of Texas, 1949. n. 4913. 